\title{
The Susceptibility Patterns of Candida Species Isolated From Urine Samples to Posaconazole and Caspofungin
}

\author{
Ali Zarei Mahmoudabadi ${ }^{1,2,} ;$ Ali Rezaei-Matehkolaei ${ }^{1,2} ;$ Fataemeh Ghanavati $^{1}$ \\ ${ }^{1}$ Department of Medical Mycology, School of Medicine, Ahvaz Jundishapur University of Medical Sciences, Ahvaz, IR Iran \\ ${ }^{2}$ Health Research Institute, Infectious and Tropical Diseases Research Centre, Ahvaz Jundishapur University of Medical Sciences, Ahvaz, IR Iran \\ ${ }^{*}$ Corresponding author: Ali Zarei Mahmoudabadi, Health Research Institute, Infectious and Tropical Diseases Research Centre, Ahvaz Jundishapur University of Medical Sciences, \\ Ahvaz, IR Iran. Tel: +98-6133330074, Fax:+98-6133332036, E-mail: zarei40@hotmail.com
}

Received: October 1, 2014; Revised: November 22, 2014; Accepted: December 5, 2014

\begin{abstract}
Background: Candiduria is a rising condition among hospitalized patients and Candida albicans is the most common recovered agent. However, non-albicans Candida species (NACs) such as C. glabrata, C. krusei, C. parapsilosis, and C. tropicalis are also important. Although most Candida species especially C. albicans are sensitive to routinely used antifungals, an increasing trend in resistance has been observed among NACs.

Objectives: The aim of the present study was to detect the susceptibility of Candida strains recovered from candiduria in hospitalized patients against posaconazole and caspofungin.

Materials and Methods: $A$ total of 120 urine samples were taken from patients hospitalized in Intensive Care Units (ICUs)(65) and urology (55) wards. All recovered yeasts were differentiated by using CHROMagar Candida medium and routine tests for identification of Candida species. Minimal inhibitory concentrations (MICs) of all isolates towards posaconazole and caspofungin were determined using the microdilution method with serial dilutions from 8 to $0.0625 \mu \mathrm{g} / \mathrm{mL}$ (posaconazole) and 4 to $0.03125 \mu \mathrm{g} / \mathrm{mL}$ (caspofungin).

Results: In total, $41.7 \%$ of urine samples were positive for Candida isolation, including C. albicans (46\%), C. glabrata (24\%), C. tropicalis (16\%) and C. krusei (14\%). The MIC of caspofungin for $90 \%$ of the tested isolates was lower than $2 \mu \mathrm{g} / \mathrm{mL}$. Furthermore, $94 \%$ of the tested isolates were inhibited by posaconazole at lower than $2 \mu \mathrm{g} / \mathrm{mL}$ after 24 hours, whereas $6 \%$ of isolates had MICs of more than $4 \mu \mathrm{g} / \mathrm{mL}$.

Conclusions:This study demonstrates the importance of Candida species in urine samples from hospitalized patients in ICUs and urology wards. It showed that both tested antifungals had excellent effects on different species of Candida, however the strains from ICUs were found to be more sensitive to caspofungin than posaconazole.
\end{abstract}

Keywords: Candida; Intensive Care Units; Posaconazole; Caspofungin

\section{Background}

There has been an increasing trend in the prevalence of candidiasis during the last two to three decades. Long stays at hospitals, antibacterial therapy, immunocompromised disease, instrument therapy, corticosteroid therapy and organ transplantation have been considered as important factors for invasive candidiasis. The urinary tract is the most common site of infection among hospitalized patients. However, the majority of urinary tract infections (UTIs) are bacterial and fungal infections account for only up to $10 \%$ (1). Although, several fungi such as saprophytic molds are associated with fungal UTIs, Candida species are the most prevalent fungal isolates (2). Candiduria is a general term for the presence of Candida species in the urinary system with different forms, asymptomatic candiduria to clinical sepsis. Candiduria is a common contamination among hospitalized patients (Intensive Care Unit (ICU) and neonatal intensive care unit (NICU)) and its mortality rate is higher among immunocompromised patients.

Candida albicans is the most common agent for candi- duria, while non-albicans Candida species (NACs) such as C. glabrata, C. krusei, C. parapsilosis and C. tropicalis are also important due to increasing resistance to antifungal agents $(3,4)$. Although most species of Candida, especially C. albicans, are sensitive to routinely used antifungals increasing resistances is observed among NACs. Resistance to fluconazole among NACs is an important problem for clinicians during therapy and prophylaxis $(3,5,6)$.

Two new antifungal agents, caspofungin (class echinocandins) and posaconazole (class triazoles), have shown excellent activities against Candida species in vitro $(7,8)$. Caspofungin (Cancidas) acts against fungal growth via inhibition of 1,3- $\beta$-D-glucan synthase. The broad-spectrum of fungicidal activity against Candida species, low side effects, and favorable drug interaction profile has made caspofungin as a first-line therapy for different types of candidiasis (9). On the other hand, posaconazole (Noxafil) is an approved triazole that is usually used for the treatment of fungal UTIs (10).

Copyright (C) 2015, Ahvaz Jundishapur University of Medical Sciences. This is an open-access article distributed under the terms of the Creative Commons Attribution-NonCommercial 4.0 International License (http://creativecommons.org/licenses/by-nc/4.0/) which permits copy and redistribute the material just in noncommercial usages, provided the original work is properly cited. 


\section{Objectives}

The aim of the present study was to detect the frequency of candiduria in patients hospitalized at the ICU and urology wards of hospitals in Ahvaz, Khuzestan, Iran. In addition, the susceptibility of recovered agents was evaluated against two new antifungals, posaconazole and caspofungin.

\section{Materials and Methods}

The study and sampling from patients was approved by the Ethics committee of the Ahvaz Jundishapur University of Medical Sciences. A total of 120 urine samples were taken from patients hospitalized at ICUs $(\mathrm{n}=65,54.2 \%)$ and urology $(n=55,45.8 \%)$ wards, including $74(61.7 \%)$ males and 46 (38.3\%) females. Samples were collected in sterile containers and immediately transferred to the medical mycology laboratory, affiliated to the Ahvaz Jundishapur University of medical sciences and then 10 $\mu \mathrm{L}$ of each urine sample was inoculated on CHROMagar Candida (CHROMagar Microbiology, Paris, France) as lawn. Cultures were aerobically incubated at $35-37^{\circ} \mathrm{C}$ for 24 - 48 hours. The colored colonies on culture media were initially determined as yeast using a light microscope. Final identification of the isolated yeasts was carried out by classical methods including germ-tube formation, growth at $42-45^{\circ} \mathrm{C}$, and microscopic morphology on Cornmeal agar (High Media, India) with 1\% Tween 80 (11).

Colony counts were also applied for all cultures and total CFU per mL was calculated. The minimal inhibitory concentrations (MICs) of the isolates against posaconazole and caspofungin (Sigma, USA) were determined for all recovered isolates according to the Clinical and Laboratory Standards Institute (CLSI) guidelines (M27A3 document) (12). In the present study 50 strains of Candida species including; C. albicans (23 strains), C. glabrata , (12 strains) C. tropicalis (8 strains) and C. krusei (7 strains) were tested for antifungals susceptibility. Briefly, for each antifungal a serial dilution from 8 to $0.0625 \mu \mathrm{g} /$ $\mathrm{mL}$ (Posaconazole) and 4 to $0.03125 \mu \mathrm{g} / \mathrm{mL}$ (Caspofungin), was prepared using RPMI 1640 (Bio IDEA, Iran) in 96-well microplates (SPL, Korea). Then a standard suspension of yeast was inoculated in each well and read using the Enzyme Linked Immunosorbent Assay (ELISA) Reader (Bio Lab, USA) at $530 \mathrm{~nm}$ wavelength after 24 and 48 hours.

\section{Results}

Primary screening by CHROMagar Candida and further conventional methods yielded the isolation of Candida strains in 50 (41.7\%) urine samples of which 28 (56\%) were from ICU (male/female = 13/15) and $22(44 \%)$ from urology wards $(M / F=10 / 12)$. Candida albicans $(n=23,46 \%)$ was the most frequently recovered isolate from both sampled wards, followed by C. glabrata ( $\mathrm{n}=12,24 \%)$, C. tropicalis ( $\mathrm{n}$ $=8,16 \%)$ and $C$. krusei $(\mathrm{n}=7,14 \%)$. As shown in Table $1 \mathrm{C}$. glabrata and C. tropicalis were mainly isolated from ICU wards. The colony counts of isolated Candida from ICU were more than $104 \mathrm{CFU} / \mathrm{mL}$ in $78.6 \%$ of positive cultures (Table 2). The results of colony count in urology ward samples are shown in Table 2 . As shown, $54.5 \%$ of the samples yielded more than $104 \mathrm{CFU} / \mathrm{mL}$.

Table 1. The Frequency of Candida Species Isolated From Intensive Care Unit and Urology Wards ${ }^{\text {a }}$

\begin{tabular}{lccc}
\hline Organisms & UrologyWard & ICU & Total \\
\hline C.albicans & $12(24)$ & $11(22)$ & $23(46)$ \\
C.glabrata & $4(8)$ & $8(16)$ & $12(24)$ \\
C. tropicalis & $1(2)$ & $7(14)$ & $8(16)$ \\
C. $k r u s e i$ & $5(10)$ & $2(4)$ & $7(14)$ \\
Total & $22(44)$ & $28(56)$ & $50(100)$ \\
\hline
\end{tabular}

${ }^{\mathrm{a}}$ Data are presented No.(\%).

\begin{tabular}{|c|c|c|c|c|c|c|}
\hline Organisms & $\leq \mathbf{5 0 0}$ & $501-1000$ & $1001-5000$ & $5001-10000$ & $>10000$ & Total \\
\hline \multicolumn{7}{|l|}{ C. albicans } \\
\hline Urology & $3(13.6)$ & $2(9.1)$ & $1(4.5)$ & $1(4.5)$ & $5(22.7)$ & $12(54.5)$ \\
\hline ICU & $0(0)$ & $0(0)$ & $4(14.3)$ & $0(0)$ & $7(25)$ & $11(39.3)$ \\
\hline \multicolumn{7}{|l|}{ C. tropicalis } \\
\hline Urology & $0(0)$ & $1(4.5)$ & $0(0)$ & $0(0)$ & $0(0)$ & $1(4.5)$ \\
\hline ICU & $0(0)$ & $0(0)$ & $1(3.6)$ & $0(0)$ & $6(21.4)$ & $7(25)$ \\
\hline \multicolumn{7}{|l|}{ C. glabrata } \\
\hline Urology & $0(0)$ & $0(0)$ & $0(0)$ & $0(0)$ & $4(18.2)$ & $4(18.2)$ \\
\hline ICU & $0(0)$ & $0(0)$ & $1(3.6)$ & $0(0)$ & $7(25)$ & $8(28.6)$ \\
\hline \multicolumn{7}{|l|}{ C. krusei } \\
\hline Urology & $0(0)$ & $0(0)$ & $1(4.5)$ & $1(4.5)$ & $3(13.6)$ & $5(22.7)$ \\
\hline ICU & $0(0)$ & $0(0)$ & $0(0)$ & $0(0)$ & $2(7.1)$ & $2(7.1)$ \\
\hline \multicolumn{7}{|l|}{ Total } \\
\hline Urology & $3(13.6)$ & $3(13.6)$ & $2(9.1)$ & $2(9.1)$ & $12(54.5)$ & $22(100)$ \\
\hline ICU & $0(0)$ & $0(0)$ & $6(21.4)$ & $0(0)$ & $22(78.6)$ & $28(100)$ \\
\hline
\end{tabular}

\footnotetext{
a Data are presented No.(\%).
} 
Zarei Mahmoudabadi A et al.

In view of susceptibility testing, $90 \%$ of isolates were sensitive to caspofungin; MIC of $0.03125 \mu \mathrm{g} / \mathrm{mL}$ for $24 \%$ of tested isolates followed by $0.0625 \mu \mathrm{g} / \mathrm{mL}$ for $16 \%, 0.125 \mu \mathrm{g} /$ $\mathrm{mL}$ for $8 \%, 0.25 \mu \mathrm{g} / \mathrm{mL}$ for $18 \%, 0.5 \mu \mathrm{g} / \mathrm{mL}$ for $16 \%, 1 \mu \mathrm{g} / \mathrm{mL}$ for $6 \%$ and $2 \mu \mathrm{g} / \mathrm{mL}$ for $2 \%$; and only $10 \%$ of isolates including two (4\%) C. glabrata and three (6\%) C. albicans were resistant to this drug (MIC $=4 \mu \mathrm{g} / \mathrm{mL}$ ). The details of MIC for each species are shown in Table 3. Posaconazole inhibited the growth of $74 \%$ of tested isolates at a concentration lower than $0.5 \mu \mathrm{g} / \mathrm{mL}$ after 24 hours, while $20 \%$ of the isolates were dose dependent ( $\mathrm{MIC}=1-2 \mu \mathrm{g} / \mathrm{mL}$ ). Resistance to posaconazole was detected in three (6\%) of the C. albicans isolates after 24 hours, however this rate increased to six (12\%) after 48 hours of incubation (Table 4 ).

\begin{tabular}{|c|c|c|c|c|c|}
\hline Susceptibility & C.albicans & C. glabrata & C. tropicalis & C. krusei & Total \\
\hline \multicolumn{6}{|l|}{ Sensitive $^{\mathrm{a}, \mathrm{b}}$} \\
\hline 0.03125 & $5(10)$ & $2(4)$ & $2(4)$ & $3(6)$ & $12(24)$ \\
\hline 0.0625 & $3(6)$ & $2(4)$ & $3(6)$ & $0(0)$ & $8(16)$ \\
\hline 0.125 & $2(4)$ & $0(0)$ & $1(2)$ & $1(2)$ & $4(8)$ \\
\hline 0.25 & $6(12)$ & $1(2)$ & $1(2)$ & $1(2)$ & $9(18)$ \\
\hline 0.5 & $3(6)$ & $5(10)$ & $0(0)$ & $0(0)$ & $8(16)$ \\
\hline 1 & $1(2)$ & $0(0)$ & $0(0)$ & $2(4)$ & $3(6)$ \\
\hline 2 & $0(0)$ & $0(0)$ & $1(2)$ & $0(0)$ & $1(2)$ \\
\hline \multicolumn{6}{|l|}{ Resistance $^{a, c}$} \\
\hline 4 & $3(6)$ & $2(4)$ & $0(0)$ & $0(0)$ & $5(10)$ \\
\hline MIC $50^{d}$ & 0.25 & 0.5 & 0.0625 & 0.125 & 0.25 \\
\hline MIC 90 & 4 & 4 & 2 & 1 & 2 \\
\hline Total $^{\mathrm{a}}$ & $23(46)$ & $12(24)$ & $8(16)$ & $7(14)$ & $50(100)$ \\
\hline
\end{tabular}

a Data are presented No. (\%).

b Concentration $(\mu \mathrm{g} / \mathrm{mL}) \leq 2$.

C Concentration $(\mu \mathrm{g} / \mathrm{mL})>2$.

$\mathrm{d}$ Abbreviation: MIC, minimum inhibitory concentration.

Table 4. Susceptibility of Candida Isolates to Posaconazole After Twenty-Four and Forty-Eight Hours

\begin{tabular}{|c|c|c|c|c|c|c|c|c|c|c|}
\hline \multirow[t]{2}{*}{ Susceptibility } & \multicolumn{2}{|c|}{ C. albicans } & \multicolumn{2}{|c|}{ C. glabrata } & \multicolumn{2}{|c|}{ C. tropicalis } & \multicolumn{2}{|c|}{ C. krusei } & \multicolumn{2}{|c|}{ Total } \\
\hline & $24 \mathrm{~h}$ & $48 \mathrm{~h}$ & $24 \mathrm{~h}$ & $48 \mathrm{~h}$ & $24 \mathrm{~h}$ & $48 \mathrm{~h}$ & $24 \mathrm{~h}$ & $48 \mathrm{~h}$ & $24 \mathrm{~h}$ & $48 \mathrm{~h}$ \\
\hline \multicolumn{11}{|l|}{ Sensitive $^{\mathrm{a}, \mathrm{b}}$} \\
\hline 0.0625 & $6(12)$ & $3(6)$ & $2(4)$ & $2(4)$ & $2(4)$ & $1(2)$ & $1(2)$ & $1(2)$ & $11(22)$ & $7(14)$ \\
\hline 0.125 & $2(4)$ & $1(2)$ & $3(6)$ & $1(2)$ & $3(6)$ & $2(4)$ & $1(2)$ & $1(2)$ & $9(18)$ & $5(10)$ \\
\hline 0.25 & $1(2)$ & $1(2)$ & $3(6)$ & $2(4)$ & $2(4)$ & $2(4)$ & $0(0)$ & $0(0)$ & $6(12)$ & $5(10)$ \\
\hline 0.5 & $7(14)$ & $6(12)$ & $1(2)$ & $0(0)$ & $0(0)$ & $1(2)$ & $3(6)$ & $3(6)$ & $11(22)$ & $10(2)$ \\
\hline \multicolumn{11}{|c|}{ Dose dependent ${ }^{\mathrm{a}, \mathrm{c}}$} \\
\hline 1 & $3(6)$ & $7(14)$ & $2(4)$ & $4(8)$ & $0(0)$ & $1(2)$ & $1(2)$ & $1(2)$ & $6(12)$ & $13(2)$ \\
\hline 2 & $1(2)$ & $0(0)$ & $1(2)$ & $2(4)$ & $1(2)$ & $1(2)$ & $1(2)$ & $1(2)$ & $4(8)$ & $4(8)$ \\
\hline \multicolumn{11}{|l|}{ Resistance $^{\mathrm{a}, \mathrm{d}}$} \\
\hline 4 & $2(4)$ & $3(6)$ & $0(0)$ & $1(2)$ & $0(0)$ & $0(0)$ & $0(0)$ & $0(0)$ & $2(4)$ & $4(8)$ \\
\hline 8 & $1(2)$ & $2(4)$ & $0(0)$ & $0(0)$ & $0(0)$ & $0(0)$ & $0(0)$ & $0(0)$ & $1(2)$ & $2(4)$ \\
\hline $\operatorname{MIC} 50^{\mathrm{e}}$ & 0.5 & 1 & 0.25 & 1 & 0.125 & 0.25 & 0.5 & 0.5 & 0.25 & 0.5 \\
\hline MIC 90 & 4 & 4 & 1 & 2 & 2 & 2 & 2 & 2 & 2 & 4 \\
\hline Total $^{\mathrm{a}}$ & \multicolumn{2}{|c|}{$23(46)$} & \multicolumn{2}{|c|}{$12(24)$} & \multicolumn{2}{|c|}{$8(16)$} & \multicolumn{2}{|c|}{$7(14)$} & \multicolumn{2}{|c|}{$50(100)$} \\
\hline
\end{tabular}

a Data are presented No. (\%).

b Concentration $(\mu \mathrm{g} / \mathrm{mL}) \leq 1$.

c $1<$ Concentration $\leq 2$.

d Concentration $(\mu \mathrm{g} / \mathrm{mL}) \geq 4$.

e Abbreviation:MIC, minimum inhibitory concentration. 
Zarei Mahmoudabadi A et al.

\section{Discussion}

The presence of Candida species in urine, candiduria, is an asymptomatic condition that results from contamination during urine collection in patients with bladder colonization or upper urinary tract infection and haematogenous spread from other sites (13). The incidence rate of candiduria is variable and several reports have shown different frequencies. It corresponds to more predisposing factors including, long stays at hospitals (especially in ICU and NICU), urinary indwelling catheters, abnormality in urinary tract, immunosuppressive therapy in immunocompromised patients, renal transplantation, broad spectrum antibacterial therapy and hemodialysis $(6,14-17)$. In addition, candiduria was commonly found in elderly people aged 85 years and older (18).

The incidence of candiduria was estimated as 25,000 cases per year in the USA during 2004 (15). Multiple studies indicate that $10-22 \%$ of hospital acquired UTIs are caused by Candida species $(14,15,19,20)$. Other studies have indicated the high rates of Candida colonization ranging from $12.7 \%$ to $70.6 \%(5,17,21)$ in urine samples of ICU patients. Similarly, $56 \%$ and $44 \%$ of our patients hospitalized in ICU and urology wards, respectively, were contaminated with different species of Candida. The higher frequency of candiduria in females from both groups of patients (ICU = $53.6 \%$ and urology ward $=54.5 \%$ ) is comparable with other studies, which concluded that females are at greater risk for development of candiduria $(4,10,17)$.

Candida albicans has historically been reported as the predominant cause of funguria, however in the two recent decades a paradigm shift occurred toward NACs, particularly C. glabrata, C. tropicalis and C. krusei (5, 14, $17,22)$. In some reports, $C$. tropicalis was ranked as the most important NACs $(3,17)$, while others reported that C. glabrata was the predominant species (16). In our study C. albicans was the predominant agent recovered from both groups, however in ICU patients, C. glabrata (28.6\%) and C. tropicalis $(25.0 \%)$ were the second and third-most frequent agents, respectively. In contrary to the study by Sobel et al. who believed that C. glabrata adapts well to some urine features like osmolality, substrate availability and $\mathrm{pH}$, in the current survey C. krusei (22.7\%) and C. glabrata (18.2\%) were the most prevalent species isolated from urine in urology ward patients (15). Kauffman et al. (4) believed that in persistent candiduria, as low as $10,000 \mathrm{CFU} / \mathrm{mL}$ may mean infection, whereas 10,000 to $\geq 100,000 \mathrm{CFU} / \mathrm{mL}$ may result colonization. In our study, colony counts of $\geq 10,000 \mathrm{CFU} / \mathrm{mL}$ were seen in $54.5 \%$ of ICU and 78.6\% of urology patients.

Caspofungin resistance in Candida species is known to be uncommon but some species e.g. C. tropicalis have been reported to be resistant. Pasquale et al. (21) documented the first case of $C$. tropicalis infection clinically resistant to caspofungin with MIC of $4 \mu \mathrm{g} / \mathrm{mL}$. Caspofungin resistances in C. parapsilosis and C. tropicalis isolates have been reported from China and Malaysia $(24,25)$. More- over, Krogh-Madsen et al. (22) revealed that caspofungin resistance could occur in C. glabrata during antifungal therapy. Our study showed that only five isolates (10\%), including three C. albicans (two isolates from urology ward and one from ICU) and two C. glabrata strains (one strain from each ICU and urology ward) were resistant to caspofungin (MIC $>2 \mu \mathrm{g} / \mathrm{mL}$ ). Non-albicans Candida species are usually resistant to most used antifungals $(3,6,10)$. Fekkar et al. (23) detected FKS gene mutations that increased the resistance of Candida species to caspofungin.

Posaconazole is a triazole antifungal with a spectrum of activity that extends to some yeasts, many saprophytic and some endemic fungi. In a murine model of hematogenous renal candidiasis posaconazole was demonstrated to be efficacious (24). It is known to be active against $C$. albicans and a variety of NACs, including C. krusei and $C$. glabrata (10). However, its in vitro activity against Candida species is not static (25). Consistent with this fact, in our study, resistance of $C$. albicans and C. glabrata isolates to posaconazole increased from $6 \%$ after 24 hours to $12 \%$ after 48 hours of incubation while no resistance was found among C. tropicalis and C. krusei strains.

The present study demonstrates the importance of Candida species in urine samples from hospitalized patients in ICU and urology wards. Our study shows that both tested antifungals had excellent effects on different species of Candida, however most strains from the ICU were more sensitive to caspofungin than posaconazole.

\section{Acknowledgements}

We thank the Jundishapur Infectious and Tropical Diseases Research Centre for their cooperation with this study. This article was extracted from an MSc thesis (Fataemeh Ghanavati).

\section{Authors' Contributions}

Ali Zarei Mahmoudabadi developed the original idea and the protocol, edited the final manuscript, and was the guarantor. Fataemeh Ghanavati contributed to the development of the data and analyzed the data. Ali Rezaei-Matehkolaei contributed to the development of the protocol and edited the draft of the manuscript.

\section{Funding/Support}

This study was extracted from an MSc thesis (Fataemeh Ghanavati) supported by a grant (No: 91131) from the Ahvaz Jundishapur University of Medical Sciences, Ahvaz, IR Iran.

\section{References}

1. Sobel JD, Kauffman CA, McKinsey D, Zervos M, Vazquez JA, Karchmer AW, et al. Candiduria: a randomized, double-blind study of treatment with fluconazole and placebo. The National Institute of Allergy and Infectious Diseases (NIAID) Mycoses Study Group. Clin Infect Dis. 2000;30(1):19-24.

2. Ahmadzadeh A, Valavi E, Shamsizadeh A, Zarei Mahmoudabadi A, Hydari M, Ahmadzadeh A. Fungal urinary tract infection in 
an infant with posterior urethral valves. Jundishapur J Microbiol. 2011;4(1):71-6.

3. Ozhak-Baysan B, Ogunc D, Colak D, Ongut G, Donmez L, Vural T, et al. Distribution and antifungal susceptibility of Candida species causing nosocomial candiduria. Med Mycol. 2012;50(5):529-32.

4. Kauffman CA, Fisher JF, Sobel JD, Newman CA. Candida urinary tract infections-diagnosis. Clin Infect Dis. 2011;52 Suppl 6:S452-6.

5. Singla N, Gulati N, Kaistha N, Chander I. Candida colonization in urine samples of ICU patients: determination of etiology, antifungal susceptibility testing and evaluation of associated risk factors. Mycopathologia. 2012;174(2):149-55.

6. Seifi Z, Azish M, Salehi Z, Zarei Mahmoudabadi A, Shamsizadeh A Candiduria in children and susceptibility patterns of recovered Candida species to antifungal drugs in Ahvaz. J Nephropathol. 2013;2(2):122-8.

7. Tobudic S, Kratzer C, Lassnigg A, Graninger W, Presterl E. In vitro activity of antifungal combinations against Candida albicans biofilms. JAntimicrob Chemother. 2010;65(2):271-4.

8. DiDone L, Oga D, Krysan DJ. A novel assay of biofilm antifungal activity reveals that amphotericin B and caspofungin lyse Candida albicans cells in biofilms. Yeast. 2011;28(8):561-8.

9. Thompson GR, 3rd, Wiederhold NP, Vallor AC, Villareal NC, Lewis JS, 2nd, Patterson TF. Development of caspofungin resistance following prolonged therapy for invasive candidiasis secondary to Candida glabrata infection. Antimicrob Agents Chemother 2008;52(10):3783-5.

10. Fisher JF, Sobel JD, Kauffman CA, Newman CA. Candida urinary tract infections-treatment. Clin Infect Dis. 2011;52 Suppl 6:S457-66.

11. Salehei Z, Seifi Z, Zarei Mahmoudabadi A. Sensitivity of Vaginal Isolates of Candida to Eight Antifungal Drugs Isolated From Ahvaz, Iran.J Microbiol. 2012;5(4):574-7.

12. Clinical and Laboratory Standards Institute... Reference method for broth dilution antifungal susceptibility testing of yeasts; Approved standard. 3 edWayne: Clinical and Laboratory Standards Institute; 2008.

13. Kauffman CA. Candiduria. Clin Infect Dis. 2005;41 Suppl 6:S371-6.

14. Zarei-Mahmoudabadi A, Zarrin M, Ghanatir F, Vazirianzadeh B. Candiduria in hospitalized patients in teaching hospitals of Ahvaz. Iran J Microbiol. 2012;4(4):198-203.
15. Sobel JD, Fisher JF, Kauffman CA, Newman CA. Candida urinary tract infections--epidemiology. Clin Infect Dis. 2011;52 Suppl 6:S433-6.

16. Bukhary ZA. Candiduria: a review of clinical significance and management. SaudiJ Kidney Dis Transpl. 2008;19(3):350-60.

17. Jain M, Dogra V, Mishra B, Thakur A, Loomba PS, Bhargava A. Candiduria in catheterized intensive care unit patients: emerging microbiological trends. Indian J Pathol Microbiol. 2011;54(3):552-5.

18. Fraisse T, Crouzet J, Lachaud L, Durand A, Charachon S, Lavigne JP, et al. Candiduria in those over 85 years old: a retrospective study of 73 patients. Intern Med. 2011;50(18):1935-40.

19. Zarei Mahmoudabadi A, Keradmand AR, Enayatollahi N. Frequency of candiduria in inpatients and outpatients in department of Urology, Golestan Hospital, Ahvaz, Iran. Iran J Kidney Dis. 2009;3(2):114-5.

20. Kobayashi CC, de Fernandes OF, Miranda KC, de Sousa ED, Silva Mdo R. Candiduria in hospital patients: a study prospective. $M y$ copathologia. 2004;158(1):49-52.

21. Pasquale T, Tomada JR, Ghannoun M, Dipersio J, Bonilla H. Emergence of Candida tropicalis resistant to caspofungin. J Antimicrob Chemother. 2008;61(1):219.

22. Krogh-Madsen M, Arendrup MC, Heslet L, Knudsen JD. Amphotericin B and caspofungin resistance in Candida glabrata isolates recovered from a critically ill patient. Clin Infect Dis. 2006;42(7):938-44.

23. Fekkar A, Dannaoui E, Meyer I, Imbert S, Brossas JY, Uzunov M, et al. Emergence of echinocandin-resistant Candida spp. in a hospital setting: a consequence of 10 years of increasing use of antifungal therapy? EurJ Clin Microbiol Infect Dis. 2014;33(9):1489-96.

24. Andes D, Marchillo K, Conklin R, Krishna G, Ezzet F, Cacciapuoti A, et al. Pharmacodynamics of a new triazole, posaconazole, in a murine model of disseminated candidiasis. Antimicrob Agents Chemother. 2004;48(1):137-42.

25. Cuenca-Estrella M, Gomez-Lopez A, Cuesta I, Zaragoza O, Mellado E, Rodriguez-Tudela JL, et al. Frequency of voriconazole resistance in vitro among Spanish clinical isolates of Candida spp. According to breakpoints established by the Antifungal Subcommittee of the European Committee on Antimicrobial Susceptibility Testing. Antimicrob Agents Chemother. 2011;55(4):1794-7. 